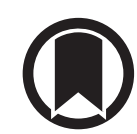

CrossMark

\section{A multi-organ loss of tissue phenotype in COPD: involvement of the functional muscle-bone unit}

\author{
To the Editor:
}

In the ECLIPSE (Evaluation of COPD Longitudinally to Identify Predictive Surrogate Endpoints) chronic obstructive pulmonary disease (COPD) cohort including 1817 men and women aged 40-75 years with full computed tomography (CT) data, CELLi et al. [1] showed that emphysema progression was associated with loss of extrapulmonary tissues such as skeletal muscle and bone. I agree with the authors that identification of the patients they proposed to name a multi-organ loss of tissue (MOLT) COPD phenotype is important because of worse clinical outcomes [1] and would also like to point out that physical activity plays a significant role in the association.

A recent update of the American Thoracic Society/European Respiratory Society statement concluded that limb muscle dysfunction is a major health problem in COPD patients and suggested that exercise was the most potent option to manage this condition [2]; in the MESA (Multi-Ethnic Study of Atherosclerosis) cohort that investigated 1435 adults aged 45-84 years without clinical cardiovascular disease, greater percentage emphysema, defined as percentage of voxels <-950 Hounsfield units on full-lung CT scans, was linked to lower habitual physical activity measured objectively by actigraphy [3]. Notably, bone, as well as skeletal muscle, normally adapts to the mechanical environment resulting from physical activity $[4,5]$. Consequently, increased and decreased physical activity would generally cause, respectively, higher and lower mass of skeletal muscle and bone, which is known as the functional muscle-bone unit [6]. From this point of view, it seems that the impairment of physical activity is significantly involved, at least partly, in the MOLT COPD phenotype.

For example, the KNHANES (Korea National Health and Nutrition Examination Survey) cohort that assessed 777 men with COPD by pulmonary functional testing and measurement of body composition by dual energy X-ray absorptiometry [7] found positive correlations between appendicular muscle mass and bone mass in the hip and lumbar spine, and the correlation between muscle mass in the arms plus legs corrected by the square of height and T-score of areal bone mineral density (BMD) was numerically higher in the hip (total hip, $r=0.461$; femoral neck, $r=0.406$ ) compared to the lumbar spine ( $r=0.304$ ), which would be compatible with functional adaptation of bone to mechanical stimulation not only from muscle contraction but also from gravitational force that is comparatively stronger in the hip than in the lumbar spine. Accordingly, many elderly patients with severe COPD are likely to have fragility fractures due to sarcopenia and osteoporosis [8].

Accumulating experimental evidence indicates that bones respond to mechanical load through their resulting elastic deformation (strain) and there are fundamental rules of mechanical strain-related stimulus $[4,5,9]$. First, the peak strain positively correlates with bone gain. Second, the strain rate is another key determinant and bones respond to dynamic, rather than static, mechanical loads. These rules can explain why exercise was inversely associated with lower areal BMD in the participants without sarcopenia but not with sarcopenia [7] because both of the peak strain and strain rate engendered by exercise would be lower in the latter. In addition, only small number of cycles of mechanical loading is enough for efficiently stimulating bone and its mechanosensitivity is restored by rest interruption between the cycles. Therefore, although COPD patients might have limitations to perform exercise, relatively short-time high-impact dynamic exercise would be recommended for their bone health.

@ERSpublications

Physical activity would be significantly involved in the multi-organ loss of tissue phenotype in COPD http://ow.ly/ez2t30jLY4E

Cite this article as: Sugiyama T. A multi-organ loss of tissue phenotype in COPD: involvement of the functional muscle-bone unit. Eur Respir J 2018; 51: 1800657 [https://doi.org/10.1183/13993003.006572018]. 
If even brief exercise is difficult, an investigational anti-sclerostin antibody (romosozumab) could be expected to become the alternative drug of mechanical strain-related stimulus in the near future. Several lines of evidence have shown that the production of sclerostin, a protein primarily secreted by osteocytes embedded in bone tissue, is increased by skeletal disuse and decreased by skeletal loading, and that treatment with an anti-sclerostin antibody can result in bone gain under conditions with impaired physical activity [10].

Nevertheless, as the authors pointed out [1], the exact mechanisms of the MOLT COPD phenotype remain unclear. Further research is needed to clarify this topic.

\section{Toshihiro Sugiyama (i)}

Dept of Orthopaedic Surgery, Saitama Medical University, Saitama, Japan.

Correspondence: Toshihiro Sugiyama, Dept of Orthopaedic Surgery, Saitama Medical University, 38 Morohongo, Moroyama, Saitama 350-0495, Japan. E-mail: tsugiym@saitama-med.ac.jp

Received: April 062018 | Accepted: April 102018

Conflict of interest: None declared.

\section{References}

1 Celli BR, Locantore N, Tal-Singer R, et al. Emphysema and extrapulmonary tissue loss in COPD: a multi-organ loss of tissue phenotype. Eur Respir J 2018; 51: 1702146.

2 Maltais F, Decramer M, Casaburi R, et al. An official American Thoracic Society/European Respiratory Society statement: update on limb muscle dysfunction in chronic obstructive pulmonary disease. Am J Respir Crit Care Med 2014; 189: e15-e62.

3 Lo Cascio CM, Quante M, Hoffman EA, et al. Percent emphysema and daily motor activity levels in the general population: multi-ethnic study of atherosclerosis. Chest 2017; 151: 1039-1050.

4 Meakin LB, Price JS, Lanyon LE. The contribution of experimental in vivo models to understanding the mechanisms of adaptation to mechanical loading in bone. Front Endocrinol 2014; 5: 154.

5 Sugiyama T. Treatment of low bone density or osteoporosis to prevent fractures in men and women. Ann Intern Med 2017; 167: 899-900.

6 Duran I, Martakis K, Hamacher S, et al. Are there effects of age, gender, height, and body fat on the functional muscle-bone unit in children and adults? Osteoporos Int 2018; 29: 1069-1079.

7 Hwang JA, Kim YS, Leem AY, et al. Clinical implications of sarcopenia on decreased bone density in men with COPD. Chest 2017; 151: 1018-1027.

8 Graumam RQ, Pinheiro MM, Nery LE, et al. Increased rate of osteoporosis, low lean mass, and fragility fractures in COPD patients: association with disease severity. Osteoporos Int 2018; in press [https://doi.org/10.1007/s00198018-4483-z].

9 Sugiyama T. Physical activity and bone health: understanding mechanical strain-related stimuli. Int J Epidemiol 2018; 47: 669-670.

10 Sugiyama T, Oda H. Osteoporosis therapy: bone modeling during growth and aging. Front Endocrinol 2017; 8: 46. 